\title{
THE LATE DIAGNOSED INTESTINAL MALROTATION BY ABDOMINAL TOMOGRAPHY IN AN ADOLESCENT
}

\author{
Esra Özçakır*, Serpil Sancar*, Gokhan Orcan**, Mete Kaya* \\ * Bursa, Health Sciences Yniversity, Yuksek İhtisas Resarch and Training Hospital, Pediatric Surgey, Bursa, Turkey \\ ** * Bursa, Health Sciences Yniversity, Yuksek İhtisas Resarch and Training Hospital, Radiology, Bursa, Turkey
}

\begin{abstract}
Introduction: Intestinal malrotation is an anomaly that often manifests in newborn and infant childhood. This anomaly, which is rarely seen in older children and adults, is usually detected incidentally during imaging studies or during laparotomy. This article is presented with the aim of emphasizing the Computarised Tomography (CT) imaging for intestinal malrotation an adolescent patient with mesenteric volvulus.

Case Report: A sixteen-year-old male patient presented with epigastric pain and bilious vomiting after feeding. In ultrasonography (US), intestinal loops were surrounding along the superior mesenteric artery (SMA) and venous (SMV) vessels tract. In CT, it was noted that SMA marks a whirl sign in the midline of the abdomen. At laparotomy, there were found $360^{\circ}$ torsion of small intestine, due to intestinal malrotation. Ladd procedure was performed.

Conclusion: In cases of intestinal malrotation, preoperative diagnosis can be difficult because of the lack of specific findings in the physical examination. The delayed diagnosis may be lead to volvulus and intestinal necrosis. The diagnosis of malrotation can brought to mind at an early age but it may be delayed in later ages. In practiced hand, the evaluation of vessels traseries by detailed US and contrast-enhanced CT are characteristics of malrotation diagnosis.
\end{abstract}

Keywords: Intestinal Malrotation, Adolescent, Computarised Tomography

\section{Introduction}

Intestinal malrotation is an anomaly, which mainly manifests itself in early life. It is generally detected incidentally during imaging studies or laparatomy. In addition, intestinal malrotation may appear with atypical symptoms at older ages [1,2]. The major complication of Intestinal malrotation is midgut volvulus, which may result in mesenteric ischemia and if not immediately intervened, cause severe mortality and morbidity $[1,2,3]$.

Although abdominal ultrasound (US) is used initially for diagnosis of malrotation, the appearance of abnormal positioning of superior mesenteric artery (SMA) and vein (SMV), whirlpool sign of SMA and duodenal distention in computerize tomography (CT) are characteristic findings for definitive diagnosis [1]. This report aim to emphasized the effectiveness of tomography (CT) to determine the diagnosis of mesenteric volvulus in adolescent patient.

\section{Case report}

A 16-year-old male patient admitted to the pediatric emergency service with the complaint of intermittent abdominal pain and bilious vomiting. In his story he had been suffering from intermittent abdominal pain, which increased for the last two weeks, accompanied by occasional bilious vomiting and described epigastric pain after eating. Physical examination indicated no abdominal distention. There was tenderness and defense on palpation of epigastric region. Rebound was not present and bowel sounds were normoactive. In the laboratory examination: $\mathrm{Hb}: 12.38 \mathrm{~g} / \mathrm{dl}$; $\mathrm{BK}$ : $14.300 / \mathrm{Mm} 3$, platelet $225.800 / \mathrm{mm} 3$. Gas distribution was observed normal on the standing direct abdominal graph (SDAG).

Abdominal ultrasonography (US) revealed a suspicious appearance of small intestine loops in the inferior part of the epigastric zone, which was located in the midline of SMA and SMV trace, encircling the trace. Also heterogeneity was observed at this level of the mesentery. These US findings could not be diagnosed definitively. A CT was then performed to assess the cause of a mechanical obstruction and to investigate it's cause. Abdominal aorta and vena cava inferior were 
normal and SMA showed a barber pole sign towards the midline of the abdomen, nearly $5 \mathrm{~cm}$ inferior from the origin of aorta (Figures 1 A, B, C, D). Also the segments of small intestine were spiraling around SMA and SMV and the 3 rd segment of the duodenum was dilated and tortuous. Angiographic CT images, performing volume rendering $3 \mathrm{D}$ reconstruction of $C T$, indicate clockwise twisting of both SMA and SMV in the corkscrew appearance (Figure 2). Because of CT findings suggested mesenteric volvulus, emergency surgery was planned.

Laparatomy was done by midline incision and verified the diagnosis. All small intestines and cecum had a single mesentery, which had undergone 360 degree torsion. No circulatory disturbance was detected. It was observed following detorsion that cecum was located on the left upper quadrant; ascending colon was adherent to duodenum, which was descending straight down, with Ladd's bands; and mesenteric veins were enlarged and lying anterior to duodenum towards the portal vein. Ladd procedure and appendectomy were carried out for the patient: The intestines were reversed on counterclockwise, Ladd's bands were excised for widening of the small intestine's mesentery, and the 3rd and 4th sections of duodenum were fixed to the retroperitoneum. Appendectomy was performed and bowels were replaced into abdomen, putting small intestines at the right side and colon to the left. The patient was discharged at the 6th day after operation without any complication. He did not have any problem during clinical follow-up. Informed consent was taken from the patient's parents for publication.

\section{Discussion}

Intestinal malrotation occurs between the 5th and 10th weeks of fetal development as a result of the failure of duodenojejunal and ileocolic segments to complete a total of 270 degrees of counterclockwise rotation around the omphalomesenteric vessels. It is defined as a high intestinal obstruction associated with bands between posterior abdominal wall and cecum which has incomplete rotation and settles on the right or left upper quadrant $[1,2,3]$. In such a situation, duodenojejunal junction is located at the right or back of SMA while cecum is located on the right lower quadrant. There may be Ladd's bands lying towards posterior abdominal wall or peritoneum, causing obstruction in duodenum [1,3].

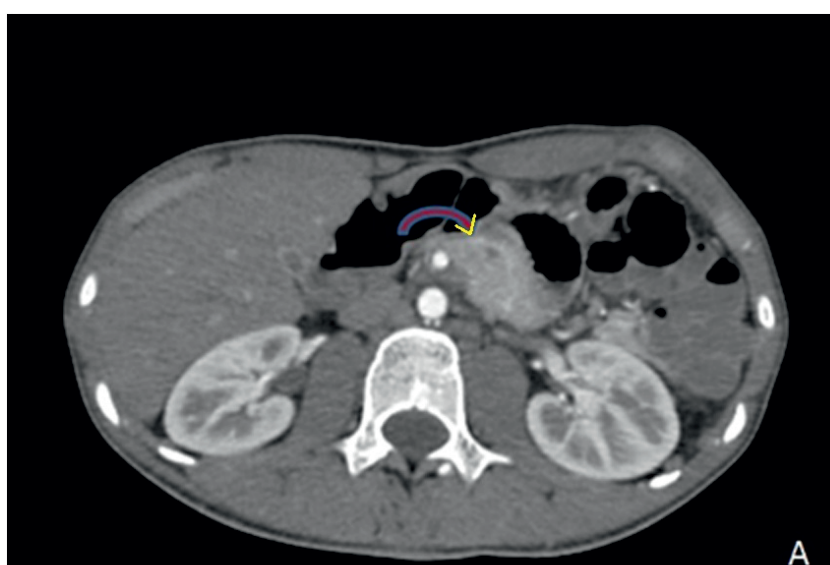

$A$

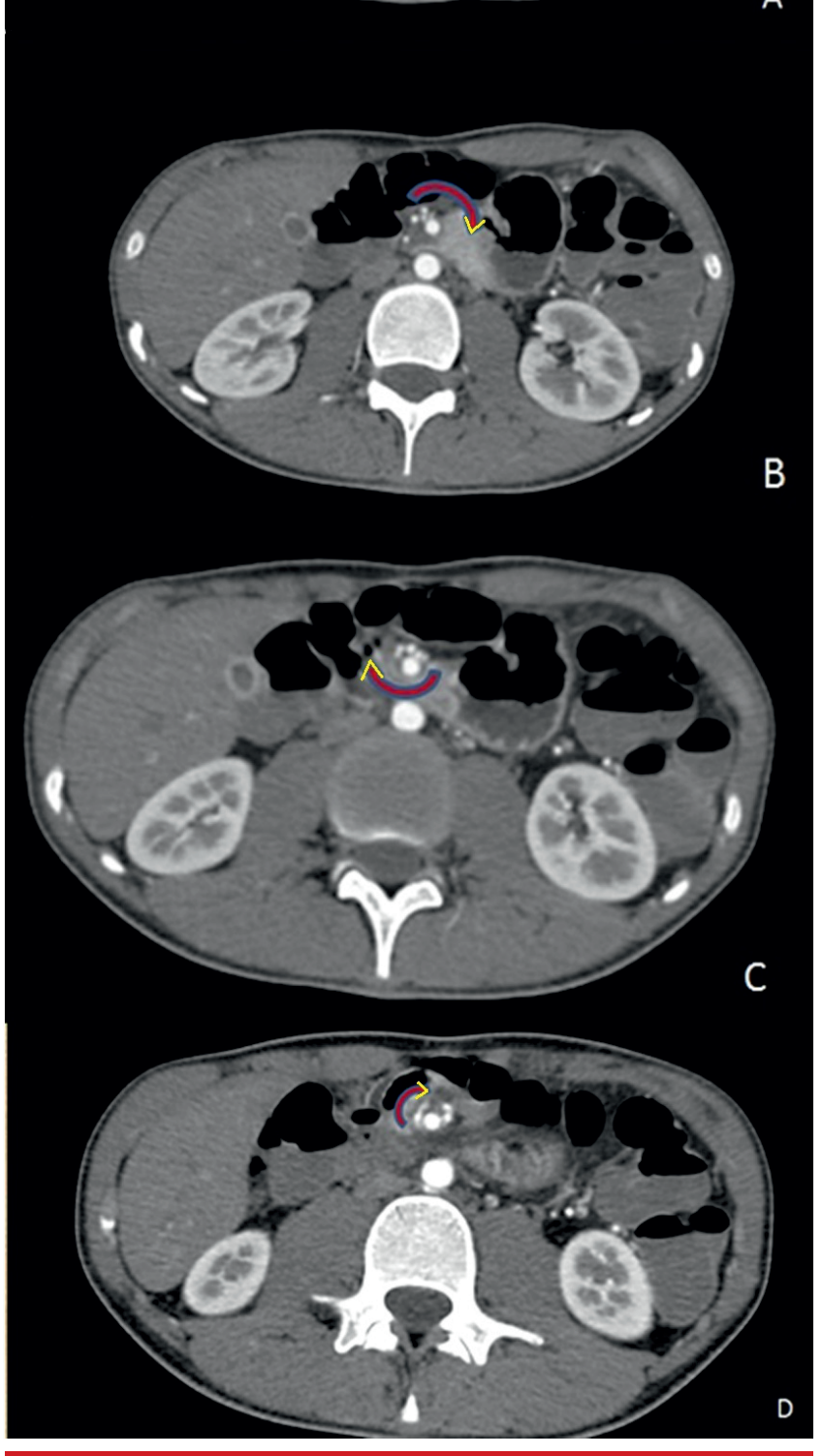

FIGURE 1. The $C T$ images (from $A$ to $D$ ) indicated whirlpool sign (arrows) with superior mesenteric vein and artery (SMV, SMA). SMA turns around superior mesenteric vein (SMV) in a clockwise fashion (direction of arrow) and abnormal rotation of the small intestine around the root of the mesentery. 


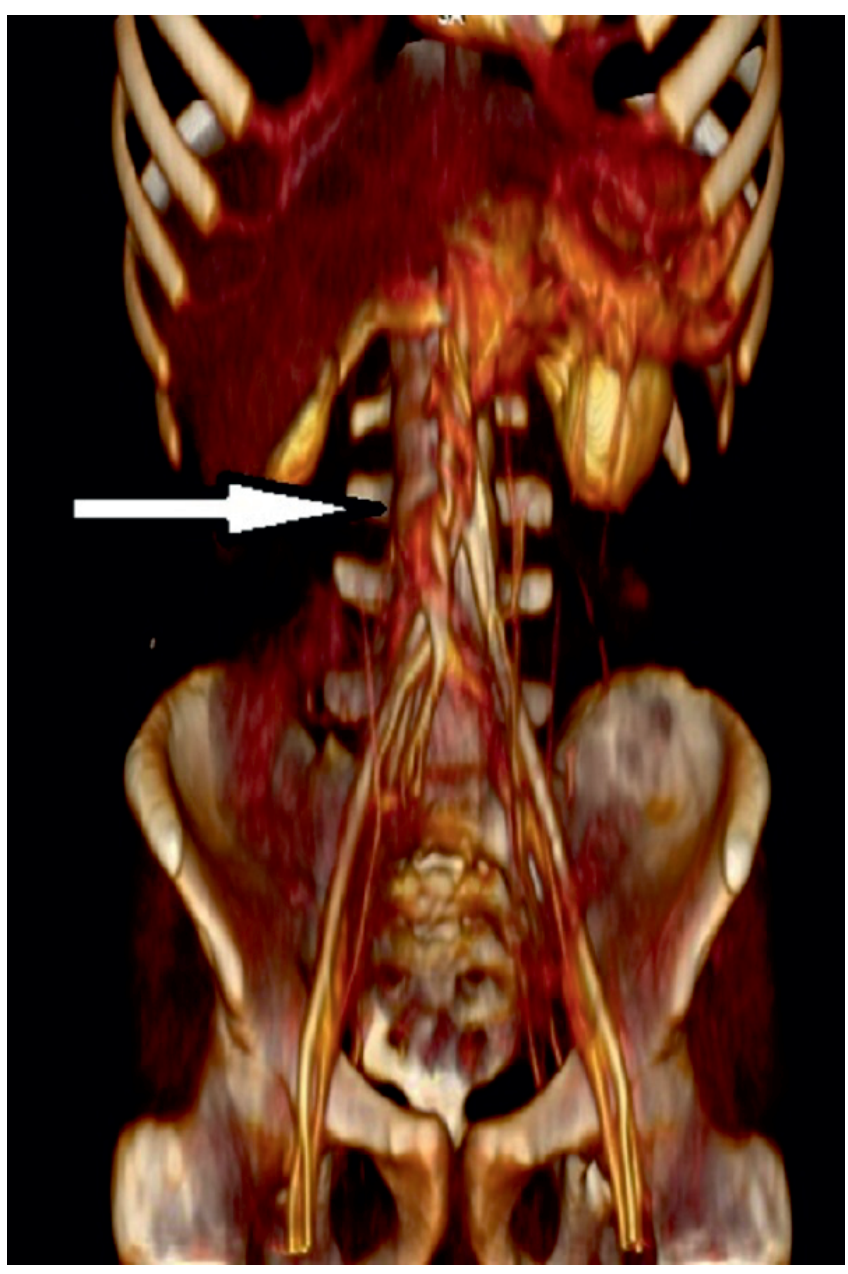

FIGURE 2. Angiographic CT image performing volume rendering 3D reconstruction, indicated clockwise twisting of both SMA and SMV in the corkscrew appearance showing the barber pole sign.

Intestinal malrotation is generally diagnosed in the neonatal period and early childhood, and it is rarely seen in older children and adults. Although it is estimated that an approximate incidence of one in 500 live births, the actual rate has not been clearly figured out, because of the abnormality may remain asymptomatic until adulthood in some cases [2,3]. Malrotation, which is reported to be less than $0.16 \%$ in adult cases, is usually detected incidentally during imaging studies or laparotomy [1,3]. Therefore our patient is valuable as the abnormality remained asymptomatic until adolescence.

Depending on the severity of malrotation, patients may be totally asymptomatic or manifest symptoms associated with obstruction, such as abdominal pain, bilious vomiting and distention [2,3]. In some cases, chyloperitoneum and acid can develop because of lymphatic obstruction. There are also researches reporting various symptoms ranging from internal hernia to mesenteric lymphocele, pneumatosis, and gastrointestinal bleeding in some patients with malrotation [4,5]. Although the cause of late presentation of malrotation symptoms (in older children and adults) has not been fully established, the severity of duodenal obstruction is thought to influence the clinical symptoms. That has been stated as the reason why some patients can live for years with no or very few symptoms $[4,6]$. In the patient's history, he had intermittent abdominal pain and finally bilious vomiting started recently, and these symptoms were compatible with intestinal obstruction. We estimate that malrotation causes to vascular compromise, and it did not cause to obstruction up to date.

The most important complication of malrotation is midgut volvulus, which can result in intestinal necrosis. Midgut volvulus is an emergency situation that causes intestines with a short mesentery to twist around the SMA axis resulting in obstruction $[1,2,3]$. Radiological imaging methods have a critical role in the diagnosis of this abnormality, which is very challenging. Although direct abdominal graphy is the preferred and frequently applied method for diagnosis, it is inadequate for the diagnosis of malrotation. Barium contrast-enhanced radiographs are highly sensitive in the diagnosis of malrotation and observing duodenojejunal junction at the right side of midline-at an abnormal position-is the most specific finding of malrotation $[1,2,3]$. Our patient's SDAG showed normal gas distribution. As intestinal obstruction was considered because of bilious vomiting and epigastric tenderness, a contrastenhanced X-ray was not performed, and the patient was evaluated with abdominal US. Abdominal US is the most commonly used non-invasive technique in the clinical evaluation of patients who have intestinal obstruction symptoms. Revealing abdominal US findings, such as a dilated duodenal segment, an abnormally located intestinal loops and the SMV is located on the left side of the SMA, may help the diagnosis in malrotating patients $[1,3,7]$. Any suspected signs in the mesentery of the small intestine were found in the abdominal US, but further imaging was needed for definitive diagnosis.

Abdominal $\mathrm{CT}$ is a relatively fast, technically simple and frequently used diagnostic method in malrotation. In tomography, the appearance of "twisted ribbon" mesentery, which surrounds SMA; collapsed status of 
intestinal loops; and a SMA image making rotations in successive sections are pathognomonic for midgut volvulus [6]. Also, the volume rendering reformat algorithm, which is present in most of the tomography devices today, easily constructs the three-dimensional structure of the tomography sections and leaves no room for doubt in the diagnosis [8]. This rotation indicated with tomographic images was first described by Buranasiri as "barber pole sign" [9]. Later on, the "clockwise" and "whirl pool sign"terms were used for the appearance of SMA on doppler US and CT as a symptom of midgud volvulus resulting from intestinal malrotation [10]. In our patient's abdominal CT, there was a barber pole sign around SMA, and our patient was considered to have mesenteric volvulus. As a result, emergency laparotomy was planned for the patient.

Ladd procedure is the conventional treatment method in malrotation abnormalities $[1,2,3]$. The procedure involves counterclockwise detorsion of the bowel, surgical division of Ladd's bands, widening of the small intestine's mesentery, appendectomy and placement of the intestines to the right of the abdomen and the colon to the left [2]. We performed Ladd procedure for our patient. After the operation, we followed-up the patient for 1 year, during which he remained asymptomatic.

In conclusion, malrotation should be considered at the first place in cases with chronic abdominal pain and vomiting. It should be also noted that abdominal $\mathrm{CT}$ is a fast and reliable diagnostic method for volvulus associated with malrotation.

\section{References}

1. Pickhardt PJ, Bhalla S. Intestinal Malrotation in Adolescents and Adults: Spectrum of Clinical and Imaging Features. AJR Am J Roentgenol, 2002; 179: 1429-35.

2. Başaklar AC, Türkyılmaz Z. Rotasyon Anomalileri. In: Abdullah Can Başaklar, Editor. Bebek ve Çocukların Cerrahi ve Ürolojik hastalıkları. Ankara: Palme Yayıncılık; 2006. p. 505-17.

3. Touloukian RJ, Smith El. Disorders of rotation and fixation. In: O'Neill JA, Rowe M, Grosfeld JL, et al, Editors. Pediatric Surgery. St Louis: Mosby; 1998. p. 1199-214.

4. Dinler G, Ceyhan M, Kalaycı AG, Rizalar R. İki buçuk yıllık karın ağrısı öyküsü olan 14 yaşında kız hasta - Ayın Olgusu. Türk Pediatri Arşivi 2008; 43(3): 105-6.

5. Belgaumkar A, Karamchandani D, Peddu P, Schulte KM. Small bowel haemorrhage associated with partial midgut malrotation in a middle aged man. World J Emerg Surg 2009; 4: 1-4.

6. Duran C, Ozturk E, Uraz S, Kocakusak A, Mutlu H, Killi R. Midgut volvulus: value of multidetector computed tomography in diagnosis. Turk J. Gastroenterol. 2008; 19: 189-92.

7. Pracros JP, Sann L, Genin G, Tran-Minh VA, Morin de Finfe $\mathrm{CH}_{\text {, }}$ Foray $\mathrm{P}$, et al. Ultrasound diagnosis of midgut volvulus: the "whirlpool" sign. Pediatr Radiol. 1992; 22(1): 18-20.

8. Horton KM, Fishman EK. The current status of multidetector row $\mathrm{CT}$ and three-dimensional imaging of the small bowel. Radiol Clin North Am. 2003; 41(2): 199-212.

9. Buranasiri Sl, Baum S, Nusbaum M, Tumen H. The angiographic diagnosis of midgut malrotation with volvulus in adults. Radiology 1973; 109: 555-6.

10. Fisher JK. Computed tomographic diagnosis of volvulus in intestinal malrotation. Radiology 1981; 140(1): 145-6. 